\title{
Lista anotada de nuevas adiciones para la flora andina de Moquegua, Perú
}

\author{
Annotated checklist of new additions to the Andean flora of Moquegua, \\ Peru
}

Nature Conservation and Plant Ecology Group. Wageningen University, Netherlands. Droevendaalsesteeg 3a, 6708PB Wageningen, The Netherlands

dbmtperu@gmail.com daniel.montesinos@wur.n
Presentado: 25/07/2012 Aceptado: $\quad$ 28/11/2012 Publicado online: 15/01/2013

\section{Daniel B. Montesinos Tubée}

\section{Resumen}

Se presentan nuevos registros de 103 especies reunidas en 79 géneros y 33 familias para la flora del norte de la cuenca alta del Río Tambo-Ichuña, en la Provincia General Sánchez Cerro en el Departamento de Moquegua. Con esto la flora de la región andina del norte de Moquegua se eleva a 507 especies, contenidas en 271 géneros y 75 familias. El número de especies endémicas se incrementó en comparación con los resultados obtenidos el 2011 por el autor. Este porcentaje de endemismo es uno de los más altos documentados para el departamento de Moquegua. Además, se señalan algunos comentarios de nomenclatura para las especies documentadas, así como colecciones de herbario revisadas y la zona geográfica donde se identificó. Esta nueva contribución al conocimiento de la flora del país es el resultado del trabajo de campo y de herbario realizados desde el año 2009 hasta el 2012, mencionando también, elementos de flora que no fueron identificadas anteriormente al nivel de especies (Montesinos, 2011). Las revisiones y comparaciones botánicas fueron realizadas en los herbarios nacionales USM y HUSA, y herbarios virtuales F y MO.

Palabras clave: Biodiversidad; florística, Moquegua, Perú.

\section{Abstract}

New records of 103 species reunited in 79 genera and 33 families are presented for the flora of the upper basin of the Tambo-Ichuña Rivers in the General Sánchez Cerro Province in the Department of Moquegua. With this study the flora of the northern Andean region of Moquegua rises to 507 species, contained in 271 genera and 75 families. The number of endemic species rises compared to the results obtained in 2011 by the author. This percentage of endemism is one of the highest documented for the department of Moquegua. Comments on nomenclature are also highlighted for the species documented as well as the herbarium collections reviewed and the geographical area where it was found. This new contribution to knowledge of the country's flora is the result of field work and herbarium reviews made from 2009 through 2012, mentioning also, elements of flora that were not previously identified to species level (Montesinos, 2011). Revisions and botanical comparisons were made at the herbaria HUSA, USM and the virtual images of F and MO.

Keywords: Biodiversity; floristic; Moquegua; Peru.

\section{Introducción}

Hasta esta publicación se conocen cinco trabajos que identifican las especies de la Flora del departamento de Moquegua: Arakaki y Cano (2001, 2003); Schwarzer et al. (2010); Montesinos $(2010,2011)$.

Una importante diversidad de especies botánicas puede encontrarse en las regiones andinas del departamento de Moquegua. Los inventarios botánicos refieren asimismo, a una elevada tasa de endemismo para Moquegua donde se pueden encontrar numerosas especies desde las lomas costeras, los desiertos, a los matorrales con cactáceas, los pastizales de Puna y las cumbres altoandinas (planicies subnivales).

Con referencia a otros departamentos del sur y colindantes con Moquegua, la diversidad florística es también muy elevada, pero se han podido encontrar diferencias en el extremo norte del departamento donde aparentemente existe una influencia climática directa del altiplano puneño y por consiguiente, más húmedo, lo cual es muy propicio para el desarrollo de una vegetación más abundante, y por lo tanto, más diversa.

Moquegua se encuentra situada dentro de las cadena montañosa volcánica del sur de Perú, la cual alberga una considerable cantidad de ecosistemas con marcadas diferencias biológicas. En tanto que la fauna, de la cual existen poquísimos registros, puede destacarse como muy importante y diversa en cuanto a especies propias de la puna.

\section{Material y métodos}

El norte de la Provincia General Sánchez Cerro en Moquegua se encuentra en los Andes del sur de Perú (vertiente del Pacífico) entre $16^{\circ} 05^{\prime} \mathrm{S}-16^{\circ} 17^{\prime} \mathrm{S}$ y $70^{\circ} 25^{\prime} \mathrm{W}-70^{\circ} 48^{\prime} \mathrm{W}$, y altitudes entre los 3400 y $4850 \mathrm{~m}$. Los estudios que representan la composición florística en el norte de Moquegua han sido realizados por Montesinos (2011), donde se registran 70 familias, 238 géneros y 404 especies. El presente trabajo adiciona 103 especies, agrupados en 79 géneros y 33 familias, concluyendo que la composición florística esta conformada por 75 familias, 271 géneros y 507 especies (en adición al trabajo de Montesinos 2011).

El norte de la región andina de Moquegua presenta una elevada tasa de especies florísticas, propias de la región andina del sur de Perú y donde se pueden encontrar numerosos endemismos (Montesinos 2011, Montesinos et al. 2012). Asimismo Schwarzer et al (2010) y Arakaki y Cano (2003) afirman que el departamento de Moquegua cuenta con un variado grupo de endemismos. 
Tabla 1. Riqueza florística total hallada para cada división taxonómica (datos de 2011 y 2012) en la cuenca alta del Rio Tambo y Rio Ichuña.

\begin{tabular}{|c|c|c|c|c|c|c|}
\hline \multirow[t]{2}{*}{ Clase } & \multicolumn{2}{|c|}{ Familias } & \multicolumn{2}{|c|}{ Géneros } & \multicolumn{2}{|c|}{ Especies } \\
\hline & 2011 & 2012 & 2011 & 2012 & 2011 & 2012 \\
\hline Pteridophytas & 10 & 1 & 17 & 1 & 23 & 2 \\
\hline Gimnospermas & 1 & - & 1 & - & 2 & 1 \\
\hline Monocots & 12 & - & 45 & 9 & 65 & 19 \\
\hline Dicots & 47 & 4 & 175 & 23 & 314 & 81 \\
\hline Subtotal & 70 & 5 & 238 & 33 & 404 & 103 \\
\hline TOTAL & 75 & & 271 & & 507 & \\
\hline
\end{tabular}

El material botánico estudiado corresponde a las colecciones realizadas por el autor en las expediciones realizadas durante los meses de marzo, abril y junio (2011) y marzo, abril y setiembre (2012). Se menciona la nomenclatura de algunos ejemplares colectados antes del 2009 que no fueron identificadas a nivel de especies en su momento. Las colecciones botánicas corresponden a diversas regiones geográficas de los distritos de Ubinas, Lloque, Yunga y Ichuña (Prov. General Sánchez Cerro) en una altura que varía entre los 3400 y los $4850 \mathrm{~m}$.

Las colectas fueron realizadas utilizando la técnica descrita por Whyte (1958), Cerrate (1969) y Young \& León (1990). Los procedimientos para la colección de plantas en campo incluyen: análisis visual del entorno geográfico y disposición de facilidades para la locación y selección de áreas geográficas a ser analizadas para la colecta de especímenes, seguidamente la colecta de especímenes vivos (de preferencia seleccionar el término de la temporada de precipitaciones para obtener un máximo en la floración vegetal que a su vez facilite la identificación). Se utilizan periódicos y prensas botánicas para la herborización y posterior secado en laboratorio. Las colectas deben contar con los órganos vegetales indispensables para la identificación taxonómica (raíces, tallos, hojas, flores y/o frutos); seguidamente, se separa el material colectado, se identifica con ayuda de claves taxonómicas, referencias bibliográficas, información sobre la distribución geográfica y posteriormente se separarán los duplicados para la entrega a diferentes herbarios, material que será de gran utilidad para futuros investigadores.

Las colectas botánicas se encuentran depositadas en los siguientes herbarios nacionales: USM, HUSA, MOL, CPUN, CUZ, HUPCH, e internacionales: WAG, MO. Las revisiones e
Tabla 2. Familias botánicas más representativas para el norte de Moquegua. Se indican el total de géneros y especies comparando los datos obtenidos el 2011 y el 2012.

\begin{tabular}{|c|c|c|c|c|c|c|}
\hline \multirow[t]{2}{*}{ Familia } & \multicolumn{3}{|c|}{ Géneros } & \multicolumn{3}{|c|}{ Especies } \\
\hline & 2011 & 2012 & Total & 2011 & 2012 & Total \\
\hline Asteraceae & 45 & 7 & 52 & 97 & 30 & 127 \\
\hline Poaceae & 22 & 6 & 28 & 33 & 13 & 46 \\
\hline Brassicaceae & 13 & - & 13 & 19 & 5 & 24 \\
\hline Malvaceae & 3 & 1 & 4 & 14 & 5 & 19 \\
\hline Caryophyllaceae & 9 & 1 & 10 & 12 & 6 & 18 \\
\hline Cactaceae & 8 & 1 & 9 & 13 & 4 & 17 \\
\hline Solanaceae & 5 & - & 5 & 14 & - & 14 \\
\hline Plantaginaceae & 2 & - & 2 & 12 & - & 12 \\
\hline Apiaceae & 8 & - & 8 & 10 & 1 & 11 \\
\hline Valerianaceae & 2 & 1 & 3 & 6 & 3 & 9 \\
\hline Amaranthaceae & 6 & - & 6 & 8 & - & 8 \\
\hline Cyperaceae & 5 & 3 & 8 & 5 & 3 & 8 \\
\hline Pteridaceae & 4 & - & 4 & 8 & - & 8 \\
\hline Iridaceae & 3 & - & 3 & 3 & 3 & 6 \\
\hline Oxalidaceae & 1 & 1 & 2 & 3 & 3 & 6 \\
\hline 63 familias & 124 & 12 & 114 & 147 & 27 & 174 \\
\hline Total (75 familias) & 238 & 33 & 271 & 404 & 103 & 507 \\
\hline
\end{tabular}

identificaciones de material botánico para el presente trabajo se llevaron a cabo comparando las colecciones de diversos autores depositados en los herbarios USM y HUSA, y las versiones virtuales en WAG, L, B, MO, F, LIL y CTES. Adicionalmente, se revisó la información taxonómica con la Base de Datos Tropicos (MO, http://tropicos.org; Peru Checklist, www.tropicos. org/Project/PEC); Base de Datos The International Plant Names Index (www.ipni.org) y Base de Datos The Plant List (www. theplantlist.org).

- Para cada especie, se indica lo siguiente:

- El nombre científico y autor.

- (Bas.) el Basónimo de la especie (en caso de haber).

- Breve descripción botánica; localidades donde fue colectada, en guion la localidad próxima y el Distrito, rango altitudinal.

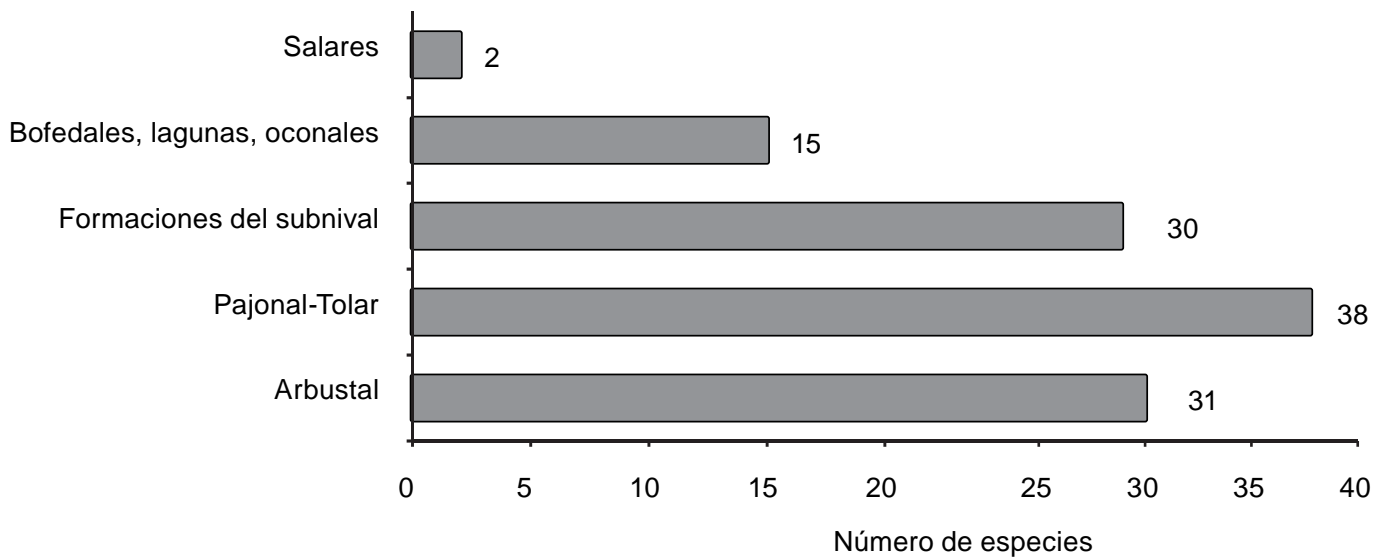

Figura 1. Nuevos registros (especies) por formaciones vegetales. 
Tabla 3. Géneros con mayor número de especies para la cuenca alta del río Tambo-Ichuña.

\begin{tabular}{|c|c|c|c|c|}
\hline Familia & Género & 2011 & 2012 & Total \\
\hline Asteraceae & Senecio & 20 & 5 & 25 \\
\hline Plantaginaceae & Plantago & 11 & - & 11 \\
\hline Malvaceae & Nototriche & 9 & 1 & 10 \\
\hline Poaceae & Calamagrostis & 5 & 4 & 9 \\
\hline Asteraceae & Werneria & 5 & 4 & 9 \\
\hline Solanaceae & Solanum & 8 & - & 8 \\
\hline Fabaceae & Astragalus & 7 & - & 7 \\
\hline Asteraceae & Perezia & 4 & 2 & 6 \\
\hline Malvaceae & Tarasa & 4 & 2 & 6 \\
\hline Valerianaceae & Valeriana & 5 & 1 & 6 \\
\hline Asteraceae & Baccharis & 5 & - & 5 \\
\hline Iridaceae & Sisyrinchium & 2 & 3 & 5 \\
\hline Brassicaceae & Lepidium & 2 & 3 & 5 \\
\hline Cactaceae & Cumulopuntia & 3 & 2 & 5 \\
\hline Oxalidaceae & Oxalis & 3 & 2 & 5 \\
\hline Asteraceae & Xenophyllum & 1 & 3 & 4 \\
\hline Poaceae & Poa & 2 & 2 & 4 \\
\hline Caryophyllaceae & Paronychia & 2 & 2 & 4 \\
\hline Asteraceae & Hypochaeris & 4 & - & 4 \\
\hline
\end{tabular}
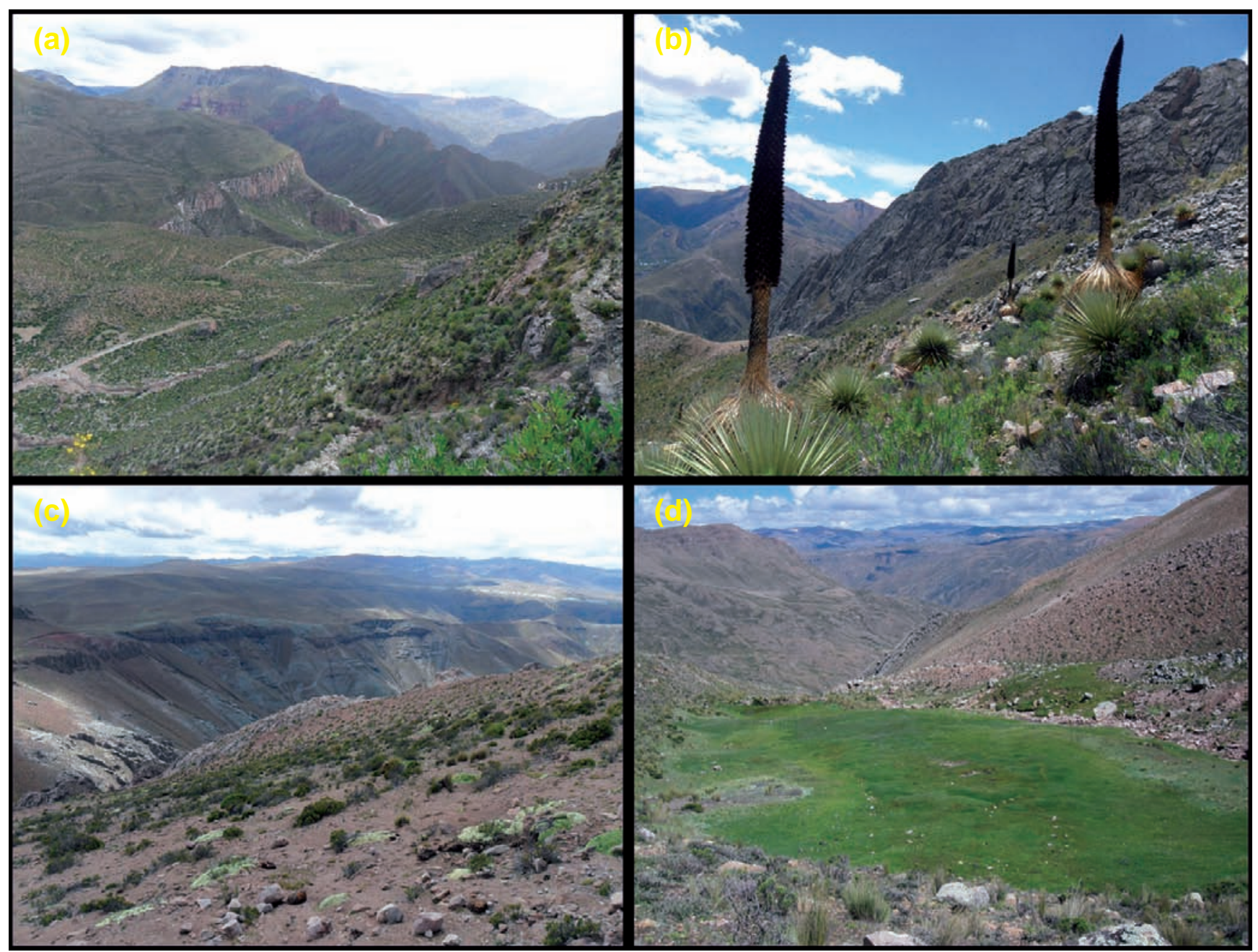

Figura 2. (a) Vista panorámica de Lucco - Exchaje. 3700 m. (b) Vista de los rodales de Puya raimondii en Ccasuyama, Yunga, $4200 \mathrm{~m}$. (c) Vista de Yanapuquio, Yanahuara, Ichuña. 4700 m. (d) Bofedales en Siliaca, Yunga. 4500 m. 


\section{Lista anotada de nuevas adiciones para la flora andina de Moquegua}

- Se incluyen 14 casos no identificados a nivel de especies.

- * Indica los duplicados entregados al herbario HSP (en formación).

\section{DIVISIÓN PTERIDOPHYTA}

ISOËTACEAE

1. Isoetes cf. boliviensis $\mathrm{U}$. Weber

Helecho acuático, sumergido. Habita lagunas ocasionales altoandinas. 4200-4250 m. Laguna Ichucochapata-Ichuña, Distrito de Ichuña.

Col.: Montesinos 2588 (USM, WAG). Rev.: Cano et al. 12967, 13011 (USM).

OphiOgLOSSACEAE

2. Ophioglossum crotalophoroides Walter

Helecho que habita pajonales húmedos y de manera inusual, en laderas arbustivas con suelos muy húmedos. 4060-4170 m. El Rancho-Tassa, Distrito de Ubinas; Ccasuyama-Siliaca, Distrito de Yunga.

Col.: Montesinos 3000 (USM, *), 3006 (USM). Rev.: Weigend et al. 8581 (USM); Beltrán 4098 (USM); León \& Young 1887 (USM).

DIVISIÓN GIMNOSPERMAS

EPHDRACEAE

3. Ephedra breana L.

Arbusto en quebradas con cactáceas y arbustos espinosos. 3800-3850 m. Distrito de Lloque.

Col.: Montesinos 3685 (USM, *). Rev.: Raimondi 863 (USM); Hutchinson 634 (USM).

DIVISIÓN ANGIOSPERMAS

\section{CYPERACEAE}

4. Phylloscirpus boliviensis (Barros) Dhooge \& Goetgh. Bas.: Scirpus boliviensis Barros

Hierba acaule. Habita pajonales continuamente húmedos. 4070 m. Patune-Camata, Distrito de Ubinas.

Col.: Montesinos 2291 (USM, HUPCH, CPUN, MO, WAG). Rev.: Salvador et al. 855 (USM).

5. Schoenoplectus californicus (C.A. Mey.) Soják Bas.: Elytrospermum californicum C.A. Mey.

Hierba acuática, en lagunas permanentes, tallos sumergidos. 4410 m. Laguna Queralacocha-Querala, Distrito de Ubinas Col.: Montesinos 3111 (USM, *). Rev.: Beltrán 6982 (USM); Aponte et al. 187 (USM).

6. Trichophorum rigidum (Boeckeler) Goetgh.

Bas.: Scirpus rigidus Boeckeler

Hierba acaule, abundante. Habita pajonales continuamente húmedos. 3800-4400 m. Distritos de Ubinas, Yunga y Ichuña. Col.: Montesinos 2982, 3047 (USM), 3029 (USM, *), 3047 (USM). Rev.: Mondragón \& Postigo 40 (USM); Smith 2791 (USM); Smith et al. 9182 (USM).

\section{IRIDACEAE}

7. Sisyrinchium brevipes Baker

Hierba anual de flores amarillas, en pajonales y laderas rocosas altoandinas. 4200-4500 m. Irupampa-Camata, Distrito de Ubinas; Yuraqcancha-Pampilla, Siliaca-Yunga, Distrito de Yunga.

Col.: Montesinos 2596, 3014 (USM). Rev.: Smith et al. 9656, 9550, 9810, 11825 (USM).

8. Sisyrinchium jamesonii Baker

Hierba anual de flores amarillas. Laderas arbustivas. 37003800 m. Ccellan-Lucco, Distrito de Lloque.

Col.: Montesinos 3766 (USM, *). Rev.: Smith et al. 10355A (MO).
9. Sisyrinchium porphyreum Kraenzl.

Hierba anual y acaule de flores rojas. Bofedales. 4500-4600 m. Coalaque, Distrito de Ubinas.

Col.: Montesinos 2392 (USM, HUPCH, MO, WAG). Rev.: Weberbauer 321 (MO).

\section{POACEAE}

10. Anthochloa lepidula Nees \& Meyen

Gramínea acaule, en laderas altoandinas. 4650-4750 m. Perusa-Yunga, Distrito de Yunga; Yanapuquio-Yanahuara, Distrito de Ichuña.

Col.: Montesinos 3791 (USM, *), 3854 (USM). Rev.: Peterson 16409 (USM); Beltrán 7086 (USM); Cano \& Valencia 10081 (USM); Smith \& Buddensiek 11200 (USM).

11. Calamagrostis brevifolia (J. Presl) Steud.

Bas.: Deyeuxia brevifolia J. Presl

Gramínea, en pajonales inundados con pastoreo. 3550-3600 m. Yinquipampa-Pampilla, Distrito de Yunga.

Col.: Montesinos 3048 (USM, MOL, ${ }^{*}$ ). Rev.: Tovar 6317 , 3422 (USM); Tovar et al. 9926 (USM); Tapia 508 (USM).

12. Calamagrostis heterophylla (Wedd.) Pilg.

Bas.: Deyeuxia heterophylla Wedd.

Gramínea, en borde de lagunas y bofedales. 4600-4650 m. Queralacocha-Querala, Distrito de Ubinas.

Col.: Montesinos 3094 (USM, *). Rev: Weberbauer 6901 (F).

13. Calamagrostis macbridei Tovar

Habita canales de agua con sulfuro. 4000-4050 m. El Rancho-Tassa, Distrito de Ubinas.

Col.: Montesinos 3001 (USM, *). Rev.: Smith et al. 10833, 12560 (USM); Cano et al. 10383 (USM); Tovar 5232 (USM).

14. Calamagrostis trichophylla Pilg.

Gramínea, en pajonales andinos. 4400-4500 m. Sura-Yunga, Distrito de Yunga.

Col.: Montesinos 3705 (USM, *). Rev.: Tovar 6175, 6281, 6702, 6819, 6843 (USM).

15. Distichlis humilis Phil.

Gramínea acaule, en flancos arenosos, suelos inundados, pajonales halófilos. 3400-3520 m. Arenal de Carabaya, Distrito de Yunga; Ccellan-Lucco, Distrito de Lloque.

Col.: Montesinos 3052 (USM, *). Rev.: Vargas 12075 (USM); Peterson et al. 14829 (USM).

16. Lolium perenne $\mathrm{L}$.

Gramínea, en bordes de caminos y áreas agrícolas. 3450-3500 m. Huraypampa-Camata, Distrito de Ubinas.

Col.: Montesinos 2994 (USM, MOL, $\left.{ }^{*}\right)$. Rev.: Peterson et al. 20620 (USM).

17. Mublenbergia fastigiata (J. Presl) Henrard

Bas.: Sporobolus fastigiatus J. Presl

Gramínea acaule, habita pajonales húmedos con pastoreo. 3900-3950 m. Crucero-Ichuña, Distrito de Ichuña. Crucero, Ichuña.

Col.: Montesinos 3838 (USM, *). Rev.: Beltrán 6398 (USM); León et al. 5653 (USM); Peterson et al. 20605 (USM); Smith et al. 10791 (USM)

18. Poa gilgiana Pilg.

Gramínea, en pajonales andinos. 4450-4500 m. Sura-Yunga, Distrito de Yunga.

Col.: Montesinos 3702 (USM, *). Rev.: Cano et al. 10492 (USM); Tovar 6423 (USM).

19. Poa spicigera Tovar

Gramínea, en laderas altoandinas. 4700-4750 m. PerusaYunga, Distrito de Yunga.

Col.: Montesinos 3793 (USM, *). Rev.: Salaverry 85 (USM); Tovar 6742 (USM); Tovar et al 9629 (USM); Roque 4968 (USM); Cerrate et al. 4722 (USM). 
20. Triniochloa stipoides (Kunth) Hitch. Bas.: Podosemum stipoides Kunth Gramínea. Habita laderas arbustivas cercanas a terrazas agrícolas. 3450-3900 m. Distritos de Yunga, Ubinas y Ichuña. Col.: Montesinos 2235 (USM, WAG). Rev.: Cano et al. 9339 (USM); Cano 3125, 3571 (USM); Cano \& Aguilar 5182 (USM).

21. Trisetum spicatum (L.) K. Richt

Bas.: Aira spicata L.

Gramínea, en pajonales altoandinos, suelos crioturbados y borde de lagunas. 4450-4800 m. Distritos de Yunga y Ubinas.

Col.: Montesinos 3707 (USM, *). Rev.: Peterson et al. 20427 (USM); Tovar 8973 (USM); Smith et al. 12111 (USM); Peterson et al. 19133 (USM); Cano et al. 7159 (USM); León \& Young 1437 (USM).

22. Vulpia myuros (L.) C.C. Gmel.

Bas.: Festuca myuros L.

Habita laderas arbustivas cercanas a terrazas agrícolas. 38503900 m. Ccellan-Lucco, Distrito de Lloque.

Col.: Montesinos 3761 (USM). Rev.: Weigend et al. 8492 (USM); Albán 6316 (USM); Cerrate et al. 6269 (USM).

\section{Division MAgnOLIOPHYTA}

\section{APIACEAE}

23. Bowlesia tropaeofolia Gillies \& Hook.

Hierba anual. Habita laderas rocosas altoandinas. 4500-4800 m. Distritos de Ubinas, Yunga y Ichuña.

Col.: Montesinos 3855 (USM, ${ }^{*}$ ). Rev.: Hutchinson 1820 (USM); King et al. 185 (USM); Iltis

et al. 131 (USM); Roque \& Ramirez 4136 (USM); Roque 4922 (USM).

\section{AsteraceAe}

24. Ambrosia artemisioides Meyen \& Walp. ex Meyen Subarbusto de capítulos amarillos, en laderas arbustivas. $3470 \mathrm{~m}$. Toreqaqa, Distrito de Lloque.

Col.: Montesinos $3783\left(\mathrm{USM},{ }^{*}\right)$. Rev.: La Torre 2315 (USM); Cáceres et al. 778 (USM);

25. Arakaki 106 (USM); Ferreyra 2577, 11672 (USM). Barnadesia pycnophylla Muschl.

Arbusto espinoso. Habita quebradas arbustivas. $3680 \mathrm{~m}$. Ccellan-Lucco, Distrito de Lloque.

Col.: Montesinos 3771 (USM, ${ }^{*}$ ). Rev.: Camino s.n. (USM); Tovar 1099 (USM); Weigend \&

Dostert 97/30 (USM); Dudley 9053 (USM).

26. Chaetanthera peruviana A. Gray

Hierba anual, capítulos blancos. Habita laderas pronunciadas y con densa vegetación arbustiva. 4100-4500 m. PatuneCamata, Distrito de Ubinas.

Col.: Montesinos 3727 (USM, *). Rev.: Beltrán 333 (USM); Cerrate \& Tovar 1757 (USM).

27. Coreopsis fasciculata Wedd.

Subarbusto de capítulos amarillos. Habita quebradas arbustivas. 3700-3800 m. Ccalo Ccalo-Tassa, Distrito de Ubinas. Col.: Montesinos 3026 (USM, MOL, *). Rev.: Cano et al. 9458 (USM); Rodriguez et al. 2202 (USM); La Torre 2354 (USM); Bernardi et al. 16489 (USM).

28. Erigeron rosulatus Wedd.

Subarbusto postrado, acaule, de capítulos rosados. $4720 \mathrm{~m}$. Choco-Choco, Distrito de Yunga.

Col.: Montesinos 3937 (USM, *). Rev.: Dillon \& Turner 1305 (F).

29. Galinsoga parviflora Cav.

Hierba anual. Habita quebradas arbustivas, áreas agrícolas y borde de caminos. 3400-3900 m. Distritos de Lloque, Yunga, Ubinas y Ichuña.
Col.: Montesinos 1001 (HUSA). Rev.: Sánchez Vega 2727, 3455 (USM).

30. Galinsoga unxioides Griseb.

Hierba anual, acaule, de capítulos amarillo-morados, en pajonales húmedos. 3750-4200 m. Yuraqcancha-Pampilla, Distrito de Yunga; Ichuña, Distrito de Ichuña.

Col.: Montesinos 2500 (USM, CUZ, MO). Rev.: Bennett 2474 (USM); Aguilar s.n. (USM).

31. Grindelia tarapacana Phil.

Arbusto de capítulos amarillos. Quebradas arbustivas. 3730 m. Altarani-Yunga, Distrito de Yunga.

Col.: Montesinos $3228\left(\right.$ USM, $\left.^{*}\right)$. Rev.: Solomon 2824 (USM); Ferreyra 2590 (USM); Gonzáles Jiménez 19 (USM).

32. Hypochaeris mucida Domke

Hierba acaule, hojas densamente pubescentes y capítulos amarillos. Laderas y cumbres rocosas semi-áridas. 3760-4500 m. Distritos de Yunga y Ubinas.

Col.: Montesinos 2041 (USM, HUPCH, MO, WAG), 3183 (USM, MOL, ${ }^{*}$ ).

33. Leucheria daucifolia (D. Don) Crisci

Bas.: Ptilurus daucifolius D. Don

Hierba anual, capítulos blancos. Habita laderas rocosas altoandinas subnivales. Yanapuquio-Yanahuara, Distrito de Ichuña. $4600 \mathrm{~m}$.

Col.: Montesinos 3841 (USM, *). Rev.: Stafford 686 (F). Pietrellini 175 (USM); Mondragón \& Postigo 23 (USM); Smith \& Escalona 10206 (USM).

34. Misbrookea strigosissima (A. Gray) V.A. Funk

Bas.: Werneria strigosissima A. Gray

Hierba anual, acaule, capítulos blancos. 4170-4500 m. Habita quebradas arbustivas con pajonales.

Col.: Montesinos 3008 (USM, MOL, *). Rev.: Franquemont 315 (F). Gonzáles 1487 (USM); Roque 4736 (USM).

35. Mniodes andina (A. Gray) A. Gray ex Hook.f. \& A.B.Jacks. Bas.: Antennaria andina A. Gray

Planta acolchonada, en laderas altoandinas subnivales. 4800 m. Perusa-Yunga, Distrito de Yunga.

Col.: Montesinos 3800 (USM, *). Rev.: Smith \& Cautivo 10296 (USM); Beltrán 7037 (USM); Cano et al. 19464 (USM).

36. Mniodes pulvinata Cuatrec.

Planta acolchonada. Habita laderas rocosas con pajonales. 4340-4600 m. Patune-Camata, Distrito de Ubinas; SuraYunga, Distrito de Yunga. Patune, Camata, Ubinas. Col.: Montesinos 3163 (USM, *). Rev.: Smith \& Buddensiek 11210 (USM); Cerrate \& Ferreyra 450/6231 (USM).

37. Perezia pinnatifida (Bonpl.) Wedd. Bas.: Chaetanthera pinnatifida Bonpl.

Hierba acaule, capítulos rosados. Habita laderas rocosas altoandinas subnivales. $4600 \mathrm{~m}$. Yanapuquio-Yanahuara, Distrito de Ichuña.

Col.: Montesinos 3844 (USM, *). Rev.: Macbride \& Featherstone 1136 (F). Tiller 865 (USM); Davis et al. 1703 (USM); Tovar et al. 9640 (USM).

38. Perezia pygmaea Wedd.

Hierba acaule, capítulos morado blancos. Habita bofedales. $4650 \mathrm{~m}$. Larsepesca-Coalaque, Distrito de Ubinas. Col.: Montesinos 2465 (USM, HUPCH, CPUN, CUZ, MO, WAG). Rev.: Tovar 1115 (USM).

39. Perezia sp.

Hierba acaule, hojas partidas; capítulos rojos. En cumbres y pajonales altoandinos. $4720 \mathrm{~m}$. Choco-Choco, Distrito de Yunga.

Col.: Montesinos 39421 (USM, *). 
40. Plagiocheilus solivaeformis DC.

Hierba acaule, habita pajonales húmedos con pastoreo. 3900-3950 m. Crucero-Ichuńa, Distrito de Ichuña. Crucero, Ichuña.

Col.: Montesinos 3837 (USM, *). Rev.: Cano et al. 11807 (USM); Cano et al. 10144, 11030, 12672 (USM).

41. Schkuhria multiflora Hook. \& Arn.

Hierba anual, capítulos amarillos. 3400-3600 m. Laderas arbustivas y terrenos agrícolas, Distrito de Yunga.

Col.: Montesinos 2151 (USM, WAG). Rev.: Solomon \& Nee 17985 (MO).

42. Senecio algens Wedd.

Subarbusto de hojas lineales y capítulos amarillos. Laderas rocosas cercanas a bofedales. $4650 \mathrm{~m}$. Sura-Yunga, Distrito de Yunga.

Col.: Montesinos 3816, 3934 (USM). Rev.: Mandon 129 (F).

43. Senecio graveolens Wedd.

Arbusto, hojas muy reducidas, capítulos amarillos. Laderas rocosas y planicies altoandinas. $4350-4750 \mathrm{~m}$. Distrito de Yunga.

Col.: Montesinos 3735 (USM, *), , 3944 (USM). Rev.: Vargas 1277 (MO).

44. Senecio humillimus Sch. Bip.

Hierba o subarbusto acaule, formando grupos acolchonados, hojas aromáticas (olor afín a Senecio nutans) y capítulos amarillos. Habita laderas rocosas y pajonales. 4350-4600 $\mathrm{m}$. Distritos de Ubinas y Yunga.

Col.: Montesinos 3089 (USM, MOL, *); 3737 (USM, *). Rev.: Solomon \& Kuijt 11498 (F).

45. Senecio klattii Greenm.

Hierba anual de hojas, tallos y filarias púrpuras; capítulos amarillos. $4500 \mathrm{~m}$. Yanapuquio-Yanahuara, Distrito de Ichuña.

Col.: Montesinos 3865 (USM, *). Rev.: Calisaya \& Quiroz 3869 (USM); Smith et al. 12335 (USM).

46. Senecio rhizocephalus Turcz.

Hierba acaule, hojas grisáceas y capítulos amarillos. Laderas con pajonales y tolares. $4600 \mathrm{~m}$. Yanapuquio-Ichuña, Distrito de Ichuña.

Col.: Montesinos 3846 (USM, *). Rev.: López Miranda 8175 (MO)

47. Senecio sp. 1

Acaule, hojas verde grisáceas con ligera pubescencia. Capítulos blancos. Planicies subnivales. $4650 \mathrm{~m}$. Cerro Pirhuani, Distrito de Ubinas.

Col.: Montesinos 3103 (USM, *).

48. Senecio sp. 2

Hierba acaule, hojas verde oscuras y glabras. Capítulos blancos. Planicies subnivales. 4800 m. Perusa-Yunga, Distrito de Yunga.

Col.: Montesinos 3805 (USM, *).

49. Senecio sp. 3

Arbusto, hojas pubescentes y capítulos amarillos. Cuevas y áreas rocosas agrestes. 4100-4500 m. Distritos de Yunga, Ubinas y Ichuña.

Col.: Montesinos 3729, 3734, 3869 (USM, *).

50. Vasquezia titicacensis (Meyen \& Walp.) S.F. Blake

Bas.: Wedelia titicacensis Meyen \& Walp.

Hierba anual, capítulos amarillos. 3400-4000 m. Habita quebradas arbustivas, borde de caminos y campos agrícolas. Distritos de Yunga y Ichuña.

Col.: Montesinos 2155 (USM). Rev.: Macbride \& Featherstone $89(\mathrm{~F})$.
51. Werneria caespitosa Wedd.

Planta acolchonada, verde oscura, hojas lineales, agudas. Capítulos blancos. Habita pajonales andinos y cumbres subnivales. 4300-4800 m. Condorwasi-Yanahuara, Distrito de Ichuña. Perusa-Yunga, Distrito de Yunga.

Col.: Montesinos 3873 (USM, ${ }^{*}$ ). Rev.: Carrillo 1316 (USM); Smith et al. 10618 (USM); Pettersson 123 (USM).

52. Werneria heteroloba Wedd.

Hierba acaule, arrosetada, de capítulos rosado-blancos. Habita bofedales y borde de lagunas. $4400 \mathrm{~m}$. Laguna Querala, Distrito de Ubinas.

Col.: Montesinos 3109 (USM, MOL, *). Rev.: Cerrate 2664 (USM); Beltrán 7109 (USM). Hjerting 1088 (F).

53. Werneria pygmophylla S.F. Blake

Planta acolchonada, verde grisácea, hojas ovadas. 4600-4700 m. Capítulos blancos. Yanapuquio-Yanahuara, Distrito de Ichuña.

Col.: Montesinos 3852 (USM, *). Rev.: La Torre 2421 (USM).

54. Werneria solivifolia Sch. Bip.

Hierba acaule, hojas compuestas. Capítulos oscuros, casi negros. Habita bofedales altoandinos. $4460 \mathrm{~m}$. YaribayaSiliaca, Distrito de Yunga.

Col.: Montesinos 3033 (USM, MOL, *).

55. Xenophyllum ciliolatum (A. Gray) V.A. Funk

Bas.: Werneria ciliolata A. Gray

Habita laderas altoandinas subnivales. Subarbusto anual, hojas moradas; capítulos blancos. 4600-4800 m. PerusaYunga, Distrito de Yunga. Yanapuquio-Yanahuara, Distrito de Ichuña.

Col.: Montesinos 3804 (USM, *). Rev.: Cerrate 2006 (USM); Cano et al. 19436 (USM); Roque 4799 (USM).

56. Xenophyllum dactylophyllum (Sch. Bip.) V.A. Funk

Bas.: Werneria dactylophylla Sch. Bip.

Subarbusto de capítulos blancos. En laderas altoandinas subnivales. 4800 m. Perusa-Yunga, Distrito de Yunga.

Col.: Montesinos 3789 (USM, ${ }^{*}$ ). Rev.: Roca Ramos 2 (USM); Hutchinson \& Tovar 4270 (USM); Raimondi 1589, 8389 (USM).

57. Xenophyllum weddellii (Phil.) Funk

Bas.: Werneria weddellii Phil.

Subarbusto de hojas lineales y capítulos blancos. Habita laderas rocosas y pajonales de altura. $4600 \mathrm{~m}$. GasahuasiQuerala, Distrito de Ubinas. Col.: Montesinos 3090 (USM).

\section{BORAGINACEAE}

58. Amsinckia calycina (Moris) Chater

Bas.: Lithospermum calycinum Moris.

Hierba anual, hirsuta, de flores amarillas. Habita matorrales arbustivo xerófilos. 3860 m. Ccellan-Lucco, Distrito de Lloque.

Col.: Montesinos 3757 (USM, *). Rev.: Cáceres 5341 (USM); Becker \& Terrones 1822 (USM); Gómez 129 (USM).

\section{BRASSICACEAE}

59. Brayopsis monimocalyx O.E. Schulz

Planta acaule; hojas en roseta, pubescentes, verde oscuras; flores simples, blanco oscuras. Laderas rocosas. 4000-4400 m. Distritos de Ubinas, Yunga y Ichuña.

Col.: Montesinos 3700 (USM). Rev.: Beck 9131 (LPB).

60. Brayopsis sp.

Hierba acolchonada, hojas en roseta y pubescentes. Pendientes rocosas pronunciadas. $4150 \mathrm{~m}$. Ccasuyama-Yunga, Distrito de Yunga.

Col.: Montesinos $3186\left(\right.$ USM, $^{*}$ ). 
61. Descurainia leptoclada Muschl.

Hierba de hojas pinnatipartidas, flores anaranjadas. Habita borde de caminos, muros de piedra, suelos perturbados. 3780 m. Ichuńa, Distrito de Ichuńa.

Col.: Montesinos 3840 (USM). Rev.: López Miranda 1063 (LIL).

62. Draba soratensis Wedd.

Hierba anual, acaule, de hojas verde claras, lanceoladas y flores blancas. Laderas rocosas, subinval. $4600 \mathrm{~m}$. YanapuquioYanahuara, Distrito de Ichuña.

Col.: Montesinos 3849 (USM, *). Rev.: Trinidad 474 (USM).

63. Draba sp.

Planta acolchonada, en densas masas; hojas en roseta, color verde grisáceo, pubescentes. Flores compuestas, blanco oscuras. Laderas rocosas, pajonales. $4480 \mathrm{~m}$. Sura-Yunga, Distrito de Yunga.

Col.: Montesinos 3720 (USM, *).

64. Lepidium bipinnatifolium Desv.

Hierba, hojas pinnatipartidas, flores blancas. Suelos perturbados, bordes de caminos, terrenos agrícolas. 3500-4500 m. Distritos de Lloque, Ubinas, Yunga y Ichuña.

Col.: Montesinos 3114 (USM, MOL, ${ }^{*}$ ), 3839 (USM). Rev.: Hutchinson 1739; Núñez 7623; Smith et al. 9653 (USM); Beltrán 75 (USM).

65. Lepidium chichicara Desv.

Hierba, hojas enteras, aserradas, flores blancas. Suelos perturbados, bordes de caminos, terrenos agrícolas. 3400-3700 m. Distritos de Lloque, Ubinas, Yunga y Ichuña.

Col.: Montesinos 3772 (USM, *). Rev.: Hastorf 146 (USM); Núnez 6671 (USM); Cevasco s.n. (USM); Smith \& Goodwin 8839 (USM).

66. Lepidium meyenii Walp.

Hierba acaule, de hojas verde oscuras a rojizas. Habita laderas rocosas, usualmente asociada a Azorella compacta. 4500-4700 m. Distritos de Yunga, Ubinas y Ichuña.

Col.: Montesinos 2367 (USM, HUPCH, CUZ, MO, WAG), 2396 (USM, HUPCH, CPUN, MO, WAG). Rev.: Chacón 17 (USM); Salas Zuluaga 204 (USM); Carrillo 20635 (USM).

67. Mancoa laevis Wedd.

Hierba anual, hojas enteras, flores blancas. Habita suelos calcificados, rocosos. $4200 \mathrm{~m}$. Tico Tico-Quimsachata, Distrito de Ubinas.

Col.: Montesinos 3229 (USM, WAG, *). Rev.: Wood 14724 (MO).

Cactaceae

68. Corryocactus aureus (Meyen) Hutchinson

Bas.: Cereus aureus Meyen

Cactus cilíndrico, postrado. Flores amarillas y frutos rojos. Habita laderas arbustivo xerófilas. 3400-3470 m. CcellanLucco, Distrito de Lloque.

69. Cumulopuntia pentlandii (Salm-Dyck) F. Ritter

Bas.: Opuntia pentlandii Salm-Dyck

Cactus de formas acolchonadas, tallos verde claros, areolas blancas y espinas blancas. En tolares y pajonales. $4200 \mathrm{~m}$. Yanahuara, Distrito de Ichuña.

70. Cumulopuntia sphaerica (Foerster) E.F. Anderson Bas.: Opuntia sphaerica Foerster Cactus globoso, segmentado. Flores amarillas. Habita laderas arbustivo xerófilas. 3400-3470 m. Ccellan-Lucco, Distrito de Lloque.

71. Lobivia maximiliana (Heyder ex A. Dietr.) Backeb. ex Rausch Bas.: Echinopsis maximiliana Heyder ex A. Dietr.

Cactus globoso, en grupos. Frutos esféricos, verde oscuros. Quebradas rocosas, pajonales, tolares. 3600-4100 m. Distritos de Yunga y Ichuña.

\section{CAmpanulaceae}

72. Lysipomia laciniata A. DC.

Hierba acaule, hojas lineal-dentadas. Habita borde de lagunas altoandinas y pajonales. $4600 \mathrm{~m}$. Laguna QueralacochaQuerala, Distrito de Ubinas.

Col.: Montesinos 3078 (USM, *). R: Cano 4567 (F). Cano 5238 (USM); Woytkowski 60 (USM); Davis et al. 1548 (USM).

\section{CARYOPHYLLACEAE}

73. Arenaria dicranoides Kunth

Hierba, acolchonada, de hojas lineales y agudas, verde claras. En laderas rocosas. $4480 \mathrm{~m}$. Sura-Ccasuyama, Distrito de Yunga.

Col.: Montesinos 3716 (USM). Rev.: Cook \& Cook 7 (MO).

74. Arenaria digyna Schltdl.

Hierba anual, flores blanco-moradas.

En laderas rocosas, arbustales, tolares, pajonales y planicies subnivales. 4000-4660 m. Distritos de Yunga, Ubinas y Ichuña. Col.: Montesinos 2175 (USM, HUPCH, MO, WAG), 2272 (USM, MO); 3823, 3847 (USM, *). Rev.: Cano \& Valencia 10126 (USM); Cerrate et al. 4772 (USM); Ferreyra 5252 (USM).

75. Cerastium nanum Muschl.

Hierba acaule, flores blancas. Habita laderas rocosas subnivales. $4500-4700 \mathrm{~m}$. Distritos de Yunga y Ichuña.

Col.: Montesinos 3091 (USM, HUPCH), 3850 (USM, *). Rev.: Weberbauer 2781 (MO).

76. Cerastium danguyi J.F. Macbr.

Hierba anual, hojas largas, pubescentes, flores blancas. Habita laderas rocosas pronunciadas con densa vegetación arbustiva. 4100 m. Patune-Camata, Distrito de Ubinas. Col.: Montesinos 3728 (USM, $^{*}$ ). Rev.: Smith \& Escalona 10191 (USM); Cano 3199, 4145 (USM); Cano et al. 7276 (USM).

77. Paronychia mandoniana Rohrb.

Hierba acaule, postrada, pubescente, de hojas verde rojizas y densas. Tolares y pajonales de altura. 3600-3800 m. Distrito de Yunga y en Italpallune, Distrito de Ichuña.

Col.: Montesinos 2567 (USM, HUPCH, MO, WAG). Rev.: Smith et al. 10473 (MO).

78. Paronychia muschleri Chaudhri

Hierba acaule, rastrera, con densas ramas cubiertas por hojas verde claras. Áreas rocosas. 3750-4500 m. Yanapuquio, Distrito de Ichuña.

Col.: Montesinos 2488 (USM, HUPCH, CPUN, CUZ, MO, WAG), 3856 (USM, *). Rev.: Núnez 7346 (MO).

79. Pycnophyllum sp.

Planta en forma de almohadillas. Habita terrenos con abundante salinidad, borde de ríos, suelos pantanosos. $3450 \mathrm{~m}$. Ccellan-Lucco, Distrito de Lloque.

Col.: Montesinos 3781 (USM, *).

80. Spergularia stenocarpa (Phil.) I.M. Johnst.

Bas: Arenaria stenocarpa Phil.

Hierba o subarbusto, hojas lineales largas, flores blancas. Habita quebradas arbustivas, borde de caminos. 3400-3800 m. Distritos de Yunga, Ubinas, Lloque y Ichuña.

Col.: Montesinos 2203 (USM, HUPCH, CUZ, CPUN, MO, WAG). Rev.: Cáceres 1631 (USM).; Cáceres et al. 1660 (USM).

81. Stellaria ovata Willd. ex Schltdl.

Hierba anual, en masas, hojas ovadas, flores blancas. Habita suelos perturbados muy húmedos. 4300-4500 m. Distritos de Yunga, Ubinas y Ichuña.

Col.: Montesinos 3709 (USM, *). Rev.: Cerrate 1188; Smith et al. 12369; Schunke 9695 (USM). 


\section{ESCALLONIACEAE}

82. Escallonia angustifolia C. Presl.

Árbol, hojas lanceoladas y flores blancas. Habita cauces de ríos. 3400-3500 m. Río Tambo-Pampilla, Distrito de Yunga. Col.: Montesinos 3732 (USM). Rev.: Gonzáles Jiménez 43 (USM); Arenas Ponce 51 (USM); Roque \& Betancourt 944 (USM); Miller 3674 (USM).

\section{EUPHORBIACEAE}

83. Euphorbia hinkleyorum I.M. Johnst.

Hierba anual, con látex, hojas verde rojizas, flores verdes. Habita quebradas arbustivas. 3770 m. Ccellan-Lucco, Distrito de Lloque.

\section{FABACEAE} Col.: Montesinos 3773 (USM).

84. Lupinus cuzcensis C.P. Smith Subarbusto de densas inflorescencias amarillas. Habita pajonales andinos. $4450 \mathrm{~m}$. Sura-Yunga, Distrito de Yunga. Col.: Montesinos 3714 (USM, *). Rev. Morales et al. 723 (USM).

85. Lupinus sp. 1

Subarbusto de flores blancas. Habita pajonales andinos. 4450 $\mathrm{m}$. Sura-Yunga, Distrito de Yunga. Col.: Montesinos 3713 (USM, ${ }^{*}$ ).

86. Lupinus sp. 2

Subarbusto de flores rosado-púrpuras. Habita quebradas arbustivas. 3770 m. Ccellan-Lucco, Distrito de Lloque. Col.: Montesinos 3765 (USM).

\section{Gentianaceae}

87. Gentianella cf. incurva (Hook.) Fabris

Bas.: Gentiana incurva Hook.

Hierba anual, flores anaranjdas. Habita laderas rocosas altoandinas y subnivales. $4660 \mathrm{~m}$. Yanapuquio-Yanahuara, Distrito de Ichuña.

Col.: Montesinos 3851 (USM, ${ }^{*}$ ). Rev.: Weberbauer 343 (MO).

88. Gentianella sp.

Hierba anual, de flores azules. Habita laderas rocosas, con Pycnophyllum. 4570 m. Sura-Yunga, Distrito de Yunga. Col.: Montesinos 3788 (USM, *).

\section{LAMIACEAE}

89. Hedeoma mandoniana Wedd.

Hierba de hojas ovadas, reducidas, flores blancas. Habita quebradas arbustivas con pajonales. $4170-4300 \mathrm{~m}$. SiliacaYunga, Distrito de Yunga; Condorwasi-Yanahuara, Distrito de Ichuña.

\section{Malvaceae} Col.: Montesinos 3010 (USM, *). Rev.: Davis et al. 1689 (F).

90. Fuertesimalva limensis (L.) Fryxell

Bas.: Malva limensis L.

Subarbusto, flores blancas. Habita flancos arenosos, suelos perturbados, quebradas arbustivas. $3520 \mathrm{~m}$. Carabaya-Yunga, Distrito de Yunga.

Col: Montesinos 3050 (USM, *). Rev.: Santa Cruz 397 (USM); Franquemont 164 (F).

91. Malva parviflora $\mathrm{L}$.

Hierba o subarbusto, flores blancas o rosadas. Habita suelos perturbados, borde de caminos, terrenos cultivados. 3450$3600 \mathrm{~m}$. Distritos de Ubinas y Yunga. Col.: Montesinos 3067 (USM, *).

Rev.: Cerrate 2397 (USM); Gamarra 665 (USM); King et al. 206 (USM); Alza 15 (USM).

92. Nototriche obcuneata (Baker f.) A.W. Hill

Subarbusto postrado, ramificado, hojas verde oscuras; flores blanco oscuras. 4600-4800 m. Perusa, Distrito de Yunga. Col.: Montesinos 3806 (USM). Rev.: Hill 1921 (MO).
93. Nototriche sp.

Planta acaule, hojas grisáceas muy reducidas, flores blancas. Habita laderas subnivales. 4700-4850 m. Cerro Pirhuani, Distrito de Ubinas. Perusa-Yunga; Distrito de Yunga.

Col.: Montesinos 38063807 (USM).

94. Tarasa operculata (Cav.) Krapov.

Bas.: Malva operculata Cav.

Subarbusto, hojas grisáceas, flores blanco oscuras con tintes lilas. Habita quebradas arbustivas y rocosas. $3640 \mathrm{~m}$. CcellanLucco, Distrito de Lloque.

Col.: Montesinos 3777 (USM, *). Rev.: Ferreyra 18300 (USM); Gamarra 545 (USM); Weigend \& et al. 8280 (USM).

95. Tarasa tenella (Cav.) Krapov.

Bas.: Malva tenella Cav.

Hierba anual, flores blancas. Habita flancos arenosos y suelos inundados. $3520 \mathrm{~m}$. Carabaya-Yunga, Distrito de Yunga. Col.: Montesinos 3049 (USM, *).

Rev.: Ferreyra 10982 (USM). Krapovickas \& Seijo 47838 (CTES).

\section{MONTIACEAE}

96. Montia fontana $\mathrm{L}$.

Hierba acuática, hojas ovadas, flotante. Habita aguas en movimiento, en bofedales. $4460 \mathrm{~m}$. Yaribaya-Siliaca, Distrito de Yunga.

Det.: Montesinos 3041 (USM, MOL, *). Rev.: Wurdack 1719

(F). Cano et al. 10508 (USM); Weigend et al. 8497 (USM).

97. Montiopsis cuminghii (Hook. \& Arn.) D.I. Ford

Bas.: Calandrinia cumingii Hook. \& Arn.

Hierba anual, hojas lineales y pubescentes, flores rojas. Habita quebradas arbustivas. $3760 \mathrm{~m}$. Ccellan-Lucco, Distrito de Lloque.

Col.: Montesinos 3760 (USM, *). Rev.: Pennell 13121 (USM); van der Werffet al. 20625 (USM); Núñez 69 (USM).

\section{OXALIDACEAE}

98. Hypseocharis pedicularifolia R. Knuth

Hierba acaule, hojas compuestas, frutos globosos, verde claros. Habita laderas arbustivo-xerófilas con influencia de ganado. 3860 m. Ccellan-Lucco, Distrito de Lloque.

Col.: Montesinos 3768 (USM, *). Rev.: Aedo \& Galán de Mera 11081 (USM).

99. Oxalis nubigena Walp.

Hierba acaule, anual, de hojas que varían de verde claro a rojo oscuro. Flores blancas. Habita laderas rocosas, cuevas con suelos muy húmedos y pajonales. 4300-4500 m. Distritos de Yunga, Ubinas, Ichuña.

Col.: Montesinos 3013, 3151, 3721, 3870 (USM); .3721 (USM, $\left.{ }^{*}\right)$. Rev.: Núnéez et al. 3887 (USM); Weigend \& Dostert 97/95 (USM); Aguilar 273 (USM); Cardich s.n. (USM).

100. Oxalis pinguiculacea R. Knuth

Hierba anual, peciolos y pedicelos largos, flores rosadas. Habita laderas rocosas y pajonales altoandinos. $4500 \mathrm{~m}$. Siliaca-Yunga, Distrito de Yunga.

Col.: Montesinos 3011 (USM, *). Rev.: Woytowski 450 (USM); Cano 3652 (USM).

\section{Polemoniaceae}

101. Microsteris gracilis (Douglas ex Hook.) Greene Bas.: Gilia gracilis Douglas ex Hook

Hierba acaule, anual; hojas lineal-lanceoladas y flores blancas. Habita quebradas arbustivas, pajonales, suelos perturbados y laderas subnivales. 3800-4750 m. Distritos de Lloque, Ubinas, Yunga y Ichuña.

Col.: Montesinos 2591 (USM, HUPCH, CUZ, MO, WAG); 3749 (USM); 3017, 3744 (USM, *), 3749 (USM). Rev.: Weigend et al. 8490 (USM); Smith et al. 9873, 9927 (USM). 


\section{Polygalaceae}

102. Monnina sp.

Hierba anual, hojas lineales y flores Habita quebradas arbustivas. 3400-3600 m. Ccellan-Lucco, Distrito de Lloque. Col.: Montesinos 3775 (USM).

\section{PortulacACEAE}

103. Cistanthe sp.

Hierba anual y suculenta de flores rojas. En quebradas arbustivas. $3820 \mathrm{~m}$. Ccellan-Lucco,

Distrito de Lloque.

Col.: Montesinos 3752 (USM, *).

\section{RANUNCULACEAE}

104. Ranunculus limoselloides Turcz.

Hierba acuática, de hojas ovadas y flotantes. En bofedales, aguas estancadas. 4300-4400 m. Occo-Querala, Distrito de Ubinas.

Det.: Montesinos 3071 (USM, *); 3112 (USM, MOL, *). Rev.: Roque \& Arana 3216 (USM); Smith 10433 (USM); Salvador et al. 502 (USM); Cano et al. 11676, 11987 (USM).

105. Ranunculus trichophyllus Chaix ex Vill.

Hierba acuática (sumergida o flotante), de hojas lineales y compuestas, y flores blancas. Habita aguas en movimiento. $4280 \mathrm{~m}$. Yuraqcancha-Pampilla, Distrito de Yunga. Col.: Montesinos 3743 (USM, *). Rev.: Beltrán 7129 (USM);

RosAcEAE Cano 12406 (USM); Petterson 334 (USM).

106. Aphanes andicola Rothm.

Hierba anual muy reducida. Habita laderas rocosas, asociado a Puya raimondii. 3900-4100 m. Ccasuyama-Yunga, Distrito de Yunga.

Col.: Montesinos 2132 (USM). Rev.: Beck 463 (LPB).

\section{RUBIACEAE}

107. Galium hypocarpium (L.) Endl. ex Griseb.

Bas.: Valantia hypocarpia L.

Hierba anual, de hojas lanceoladas, densas. Flores blancas. Habita laderas rocosas y quebradas arbustivas. 3800-4200 m. Distritos de Lloque, Ubinas y Yunga.

Col.: Montesinos 2143 (USM, HUPCH, MO, WAG). Rev.: Hutchinson \& Bennett 4745 (USM); Cerrate et al. 677 (USM); Ferreyra 6481 (USM); Tiller/Maas 377 (USM).

\section{SAXIFRAGACEAE}

108. Saxifraga cf. magellanica Poir.

Planta en forma de almohadillas. Hojas arrosetadas, flores verde blancas. Habita laderas rocosas subnivales. $4600 \mathrm{~m}$. Yanapuquio-Yanahuara, Distrito de Ichuña.

Col.: Montesinos 3845 (USM, *). Rev.: Smith et al. 11605; Sagástegui et al. 15777 (F). Roque \& Arana 2171 (USM); Cerrate et al. 4900 (USM).

SCROPHULARIACEAE

109. Veronica persica Poir.

Hierba anual, de flores moradas. Habita terrenos agrícolas, suelos perturbados, muros de piedra. 3500-3600 m. AltaraniYunga, Distrito de Yunga.

Col.: Montesinos 3057 (USM, MOL, *); 3066 (USM). Rev.: Bennett 1917 (USM); Roque \& Arana 1820 (USM); Llatas Quiroz et al. 3726 (USM).

TROPAEOLACEAE

110. Tropaeolum tuberosum R. \& P.

Hierba trepadora, hojas lobadas, flores anaranjadas. Habita quebradas arbustivas y terrenos agrícolas. $3600-3800 \mathrm{~m}$. Ccellan-Lucco, Distrito de Lloque; Tassa, Distrito de Ubinas. Col.: Montesinos 3776 (USM, *). Rev.: Cano 2513 (USM); Yarupaitán \& Albán 777 (USM); Meza 21 (USM).

\section{URTICACEAE}

111. Parietaria debilis G. Forst.

Sin.: Freirea debilis (G. Forst.) Jarm.

Hierba anual, hojas oblongas, flores blancas. Habita borde de campos agrícolas. $3620 \mathrm{~m}$. Yinquipampa-Pampilla, Distrito de Yunga.

Col.: Montesinos 3746 (USM, *). Rev.: Ferreyra \& Cerrate 16535; Aponte et al. 2; La Torre et al. 3110 (USM).

VALERIANACEAE

112. Aretiastrum cf. aschersonianum (Graebn. ex Weberb.) Graebn. Bas.: Valeriana aschersoniana Graebn. ex Weberb. Subarbusto, hojas muy resinosas. Habita suelos rocosos, en compañía de Azorella compacta. $4480 \mathrm{~m}$. Carmen Chaclaya, Distrito de Ubinas.

Col.: Montesinos 3154 (USM, *). Rev.: Maas 573; (USM) Cáceres 2847 (USM); Iltis \& Ugent 1487 (USM).

113. Stangea wandae Graebn.

Hierba acaule, hojas verde oscuras con hendiduras. Flores marrón violetas. Habita laderas altoandinas, subnivales. 4600-4750 m. Distritos de Ubinas, Yunga y Ichuña.

Col.: Montesinos 3097 (USM, *), 3809 (USM). Rev.: Solomon \& Kuijt 11498 (F). Weberling s.n. (USM); Tovar 1139 (USM).

114. Valeriana cf. pinnatifida Ruiz \& Pav.

Hierba acaule, hojas pinnatipartidas, flores lilas. Habita laderas rocosas con pajonales de altura. $4600 \mathrm{~m}$. GasahuasiQuerala, Distrito de Ubinas.

Col.: Montesinos 3086 (USM). Rev.: Cano 7892 (USM); Delgado \& Collado 3715 (USM); Albán 5701 (USM).

\section{VERBENACEAE}

115. Phyla nodiflora (L.) Greene Bas.: Verbena nodiflora L.

Hierba anual, de flores lilas. Habita terrenos agrícolas. 3500$3650 \mathrm{~m}$. Altarani-Yunga, Distrito de Yunga.

Col.: Montesinos 3058 (USM, *). Rev.: Cerrate et al. 4355 (USM); Huapalla 26 (USM); Blanchard et al. s/n. (USM).

\section{VIOLACEAE}

116. Viola micranthella Wedd.

Hierba anual acaule, flores blancas. Habita laderas rocosas y arbustivas, pajonales de altura. Distritos de Yunga y Ubinas. 4000-4500 m.

Col.: Montesinos 3019a (USM, *). Rev.: Mandon 942 (MO). Beltrán 208 (USM); Gutte \& Müller 9173 (USM); Tovar 916 (USM).

VIVIANACEAE

117. Balbisia meyeniana Klotzsch Arbusto, hojas lineales y flores amarillas. Habita quebradas arbustivas. 3400-3700 m. Ccellan-Lucco, Distrito de Lloque. Col.: Montesinos 3748 (USM, *). Rev.: Weberbauer 5509, 7430 (USM); Dillon et al. 3333 (F).

\section{Conclusiones y discusión}

Los resultados dan a conocer que la riqueza florística en la cuenca alta de los rio Tambo-Ichuña es más elevada de lo que inicialmente se pensó (Montesinos 2011). Con el presente trabajo se dan a conocer 103 especies reunidas en 79 géneros y 33 familias. Complementando los datos publicados el 2011 por el autor, los resultados arrojan la existencia total de 75 familias con 271 géneros y 507 especies. Aunque estos valores son significativamente elevados y caracterizan casi la totalidad la composición florística del norte de Moquegua, aún faltan zonas que explorar y se espera que este número aumente.

Asimismo, se presentan 13 casos no identificados a nivel de especies, sumado a los casos sin determinación del 2011 dan 
un total de casi 50 especies no determinadas por diversas causas (principalmente por la falta de información en herbarios). Se espera que diversas novedades botánicas se encuentren dentro de este grupo próximamente. Los complejos botánicos están referenciados a los siguientes géneros: Senecio (5 casos), Lupinus (4 casos), Gentianella (4 casos), entre otros.

Complementando los presentes datos y los publicados por Montesinos (2011), la familia que presenta la mayor riqueza florística es Asteraceae con un total de 127 especies (52 géneros), seguido de Poaceae con 46 especies (28 géneros), Brassicaceae con 24 especies (13 géneros), Malvaceae con 19 especies ( 4 géneros), Caryophyllaceae con 18 especies (10 géneros) y Cactaceae con 17 especies (9 géneros).

Entre los géneros que presentan la mayor representatividad en la cuenca alta del río Tambo-Ichuña se mencionan: Senecio (Asteraceae) con 25 especies; Plantago (Plantaginaceae) con 11 especies; Nototriche (Malvaceae) con 10 especies; Calamagrostis (Poaceae) y Werneria (Asteraceae) con 9 especies cada una; Solanum (Solanaceae) con 8 especies; Astragalus (Fabaceae) con 7 especies; Perezia (Asteraceae) y Tarasa (Malvaceae) y Valeriana (Valerianaceae) con 6 especies cada una.

En el presente trabajo se adicionan 5 nuevas familias que no habian sido reportadas anteriormente: Ophioglossaceae (Ophioglossum crotalophoroides), Montiaceae (Montia fontana y Montiopsis cuminghii), Polygalaceae (Monnina sp.), Tropaeolaceae (Tropaeolum tuberosum, en estado silvestre) y Vivianiaceae (Balbisia meyeniana).

En cuanto al hábito de los nuevos registros: 1 especie arbórea $(0,8 \%) 18$ arbustivas $(15,4 \%), 10$ almohadillas $(8,5 \%), 4$ suculentas (3,4\%), 69 herbáceas (59\%), 13 gramíneas $(11,1 \%)$ y 2 helechos $(1,7 \%)$.

Los nuevos registros corresponden a diferentes tipos de formaciones vegetales en la zona estudiada se muestran en la Figura 1, donde: 31 especies propias de laderas arbustivas $(3400-3800$ $\mathrm{m}) ; 38$ especies en tolares y pajonales de altura $(3800-4500 \mathrm{~m})$; 30 especies de áreas subnivales ( $4500-4850 \mathrm{~m}) ; 15$ especies de bofedales, lagunas y oconales $(4000-4800 \mathrm{~m})$; finalmente, 2 especies de ambientes halófitos $(3400 \mathrm{~m})$.

\section{Agradecimientos}

El autor agradece al herbario USM como institución cooperante, así como a la DGFFS del Ministerio de Agricultura por los diversos permisos de colecta. A los directores, curadores e investigadores de los siguientes herbarios por haber facilitado información y acceso a sus colecciones y por sus valiosas sugerencias en determinaciones: A. Granda, M. Flores (MOL), V. Quipuscoa (HUSA), I. Sánchez-Vega (CPUN), S. Castillo, H. Beltrán, M.I. La Torre, J. Campos, M. Chanco, H. Montoya, W. Ramírez y J. Roque (USM), I. Al-Shehbaz, J. Pruski (MO), M. Dillon (F), C. Hughes (Z), J. Wieringa (WAG), E. Urtubey (SI), C. Lehnebach (WELT), Stephan Beck (LPB), Lilian Eggers (UFRGS). Mi agradecimiento a A. Cleef (UVA-AMD), K. Sýkora (WUR) y G. Valcárcel por sus comentarios y sugerencias. Asimismo, a los colaboradores en las salidas a campo, las autoridades y población de las diferentes localidades visitadas en la Provincia General Sánchez Cerro.

\section{Literatura citada}

APG 2003. An update of the Angiosperm Phylogeny Group classification for the orders and families of flowering plants: APG II. Botanical Journal of the Linnean Society, 141: 399-436.

Arakaki M. \& A. Cano. 2001. Vegetación y estado de conservación de la cuenca del Río Ilo-Moquegua, Lomas de Ilo y áreas adyacentes. Arnaldoa 8(1): 49-70 pp.

Arakaki M. \& A. Cano. 2003. Composición florística de la cuenca del río Ilo-Moquegua y lomas de Ilo, Moquegua. Rev. peru. biol. 10(1): 5-15 pp.

Cerrate E. 1957. Notas sobre la Vegetación del Valle de Chiquian, Anuario de la Estación Altoandina de Biología. 1: 9-15 pp.

León B., J. Roque, C. Ulloa Ulloa, et al. 2006. Libro Rojo de las Plantas endémicas del Perú. Revista Peruana de Biología, Edición Especial 13(2): 1-971 pp.

Montesinos D. 2010. Cactus y Suculentas de Moquegua. Revista Quepo, Lima, Peru. Vol. 24: 29-41.

Montesinos D. 2011. Diversidad Florística de la cuenca alta del rio Tambo-Ichuña, Moquegua, Perú. Rev. peru. biol. 18(1): 119-132.

Montesinos D., A. Cleef \& K. Sýkora. 2012. Andean shrublands of Moquegua, South Peru: Prepuna plant communities. Phytocoenologia, 42 (1-2): 29-55.

Schwarzer C., F. Cáceres, A. Cano, M.I. La Torre \& M. Weigend. 2010. 400 years for long-distance dispersal and divergence in the northern Atacama Desert and Insights from the Huaynaputina pumice slopes of Moquegua, Peru. Journal of Arid Environments 74(11): 1540-1551.

Whyte R.O. 1958. Plant exploration, collection and introduction. FAO Agricultural Sctudies 1958. $117 \mathrm{pp}$

Young K.R. \& B. León. 1990. Catalogo de las plantas de la zona alta del Parque Nacional Rio Abiseo, Peru. Publ. Mus. Hist. Nat. Javier Prado, B no.34: 1-37. 